\title{
Efectos del género y la educación en la felicidad en adultos argentinos
}

\section{Effects of gender and education on happiness in argentinian adults}

\author{
Sofía Esmeralda Aunéa, ${ }^{\mathrm{a}}$, Facundo Juan Pablo Abal ${ }^{\mathrm{a}}$, Horacio Félix Attorresi ${ }^{\mathrm{a}}$ \\ anstituto de Investigaciones, Facultad de Psicología, Universidad de Buenos Aires; \\ CONICET, Argentina
}

\section{Resumen}

La felicidad se conceptualiza como una emoción positiva centrada en el presente, muy deseable pero difícil de conseguir. Para estimarla se aplicó la versión argentina de la Escala de Felicidad de Lima de Alarcón (2006) compuesta de tres dimensiones: Sentido de la Vida Perspectiva Externa (SVPE), Sentido de la Vida Perspectiva Interna (SVPI) y Satisfacción con la Vida (SCV). Se seleccionaron tres muestras independientes de 200 individuos cada una ( $50 \%$ mujeres) considerando diferentes niveles educativos -desde secundario completo hasta universitario completo-. Se realizó un análisis de la varianza (ANOVA) de dos vías en cada dimensión considerando como factores fijos el género y el nivel educativo. En las tres dimensiones la interacción entre tener un mayor nivel educativo y ser del género femenino favoreció en forma significativa los mayores puntajes de felicidad (SVPE, $\mathrm{F}=4.14, p=.02, \eta^{2}$ parcial $=.014$; SVPI, $\mathrm{F}=2.34, p=.09, \eta^{2}$ parcial $=.008 ; \mathrm{SCV}, \mathrm{F}=3.34, p=.04$, $\eta^{2}$ parcial $\left.=.011\right)$. En SVPE y SVPI un mayor nivel educativo por sí mismo tuvo como efecto puntajes de felicidad más altos (SVPE, $\mathrm{F}=4.12, p=.02, \eta^{2}$ parcial $=$ .014 ; SVPI, $\mathrm{F}=4.24, p=.02 ; \eta^{2}$ parcial $=.014$ ), mientras que en SCV fue pertenecer al género femenino el factor que aumentó en forma significativa los niveles de felicidad $\left(\mathrm{F}=3.03, p=.08, \eta^{2}\right.$ parcial $\left.=.005\right)$.

Palabras clave: felicidad, nivel educativo, género, psicología positiva.

Para citar este artículo:

Auné, S., Abal, F., \& Attorresi, H. (2017). Efectos del género y la educación en la felicidad en adultos argentinos. Liberabit, 23(2), 169-188. doi: 10.24265/liberabit.2017.v23n2.02

\begin{abstract}
Happiness is defined as a positive emotion focusing on the present, which is highly desirable but difficult to achieve. To estimate the level of happiness in adults, we used the Argentinian version of the Happiness Scale of Lima (EFL, Alarcón, 2006). The EFL measures three dimensions: External Perspective of Sense of Life (SVPE), Internal Perspective of Sense of Life (SVPI) and Life Satisfaction (SCV). Three independent samples of 200 individuals each (50\% female) were selected considering different educational levels (from high school graduates to college graduates). A two-way analysis of variance (ANOVA) was applied to each dimension taking into account gender and educational level as fixed factors. In all three dimensions, the interaction between having a higher educational level and being female favored significantly the highest scores of happiness (SVPE, $\mathrm{F}=$ 4.14, $p=.02$, partial $\eta^{2}=.014$; SVPI, $\mathrm{F}=2.34, p=.09$, partial $\eta^{2}=.008$; SCV, F $=3.34, p=.04$, partial $\eta^{2}=.011$ ). A higher level of education by itself resulted in SVPE and SVPI higher happiness scores (SVPE, $\mathrm{F}=4.12, p=.02$, partial $\eta^{2}=.014$; SVPI, F $=4.24, p=.02$; partial $\eta^{2}=.014$ ), whereas being female was the factor that significantly increased the levels of SCV happiness $(\mathrm{F}=3.03, p=.08$, partial $\left.\eta^{2}=.005\right)$.
\end{abstract}

Keywords: happiness, educational level, gender, positive psychology.

Este es un artículo Open Access bajo la licencia Creative Commons Atribución-NoComercial-CompartirIgual 4.0

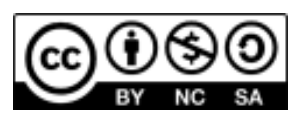


Si bien el término felicidad se ha utilizado como sinónimo de calidad de vida y bienestar, comprende aspectos más específicos (Lyubomirsky \& Layous, 2013; Veenhoven, 2015). La felicidad es una meta última desde que es un fin en sí misma (Rojas, 2016). Alcanzar la felicidad motiva la acción humana: las personas realizan decisiones y acciones que creen contribuirán a alcanzar una mayor felicidad. Decisiones como dónde vivir, con quién casarse, qué carrera estudiar, a quién votar, o si emigrar o no, son realizadas sobre la base de una expectativa de felicidad.

La felicidad es entendida como una experiencia vital propia de la condición humana. Consta de experiencias sensoriales, afectivas y evaluativas de sentirse bien y realizar una síntesis acerca de qué tan bien transcurre la vida (Rojas, 2016). Un cuarto tipo de experiencia es la mística, que se asocia con experiencias espirituales, pero no ha sido frecuentemente estudiada.

En cuanto a las experiencias sensoriales, el bienestar se asocia de forma positiva con el placer a través de los cinco sentidos y de forma negativa con el dolor. Asimismo, el bienestar se asocia directamente con emociones positivas como el amor $\mathrm{y}$ en forma inversa con emociones negativas como odio, miedo y envidia. Las experiencias evaluativas involucran una valoración acerca de cómo se desarrolla la vida de acuerdo a estándares y aspiraciones. La experiencia de logro emerge cuando se alcanzan logros y aspiraciones, lo cual favorece el bienestar. Por el contrario, la experiencia de fracaso se relaciona con no haber podido ser capaz de cumplir las metas propuestas y se asocia negativamente con el bienestar. Los cuatro tipos de experiencia se pueden complementar entre sí, generando una vivencia global y positiva de bienestar (Rojas, 2016).

Se ha definido a la felicidad asimismo como la apreciación de la vida en su conjunto, siendo condiciones adversas contra ella la pobreza, soledad y enfermedad (Veenhoven, 1998). Las personas más felices tienen sistemas inmunes más fortalecidos, mayores ingresos, parejas más estables e ideas más creativas (Lyubomirsky, King, \& Diener, 2005). Asimismo, son más extrovertidos (Andrés, Castañeiras, \& Richaud de Minzi, 2014) y tienden a ser más prosociales (Auné, Blum, Abal, Lozzia, \& Attorresi, 2014) y empáticos (Auné, Abal, \& Attorresi, 2016a). La felicidad es una emoción positiva centrada en el presente, muy deseable pero difícil de conseguir.

Generalmente se ha concebido que las personas viven típicamente mejor en países más ricos, modernos, con mayor libertad política, económica e individual; con equidad de género y calidad institucional (Veenhoven, 2015). Por el contrario, por ejemplo, la inequidad en los ingresos está asociada con efectos negativos en las oportunidades económicas y la movilidad social (Stiglitz, 2012), la salud psíquica (Wilkinson \& Pickett, 2009) y la felicidad (Oishi, Kesebir, \& Diener, 2011).

La psicología positiva ha ahondado en la temática de la felicidad sobre sus motivaciones, así como la potenciación de los aspectos positivos de la personalidad para que el ser humano pueda alcanzar un estado óptimo (Dzhidaryan, 2013; Seligman, 2009). Para Seligman, Parks y Steen (2004) existen tres formas de encontrar felicidad. La primera es el sentimiento positivo hacia el pasado, presente o futuro. La segunda ruta es la persecución de la gratificación. Esta involucra al individuo plenamente y requiere cualidades como creatividad, inteligencia social, sentido del humor y perseverancia. La tercera ruta se refiere a utilizar las propias fortalezas para contribuir con un fin social mediante el conocimiento, la bondad, la familia, la comunidad, la justicia o un poder espiritual.

Algunas investigaciones centran el estudio de la felicidad en los eventos vitales experimentados por el individuo. En esta línea, se ha hallado que los individuos son capaces de afrontar un evento vital crítico, pero si son enfrentados con dos o más eventos 
vitales de este tipo dentro de un período de cinco años, su felicidad decrece en forma significativa (Grob, 1995). En este marco, una inquietud vigente es si la frecuencia o la intensidad del afecto positivo, o ambas conjuntamente son necesarias o suficientes para la felicidad. Diener, Sandvik y Pavot (2009) argumentan que los investigadores de la felicidad deberían observar primariamente la frecuencia de la experiencia emocional positiva versus la negativa. Se basan en que la frecuencia de emociones positivas puede ser medida de forma precisa y confiable. Además, la frecuencia relativa del afecto positivo versus el negativo es tanto necesaria como suficiente para producir el estado llamado felicidad, mientras que la intensidad de la experiencia positiva no lo es. Por el contrario, Susniene y Jurkauskas (2015) argumentan que es justamente la intensidad de la experiencia lo esencial de la felicidad, y sería esta característica la que la distingue del bienestar y la satisfacción.

Para otros investigadores, los factores sociales son los que tienen el mayor impacto en la felicidad. En este sentido, Bartolini, Bilancini y Pugno (2013) realizaron un estudio longitudinal hallando que en estadounidenses el nivel de conexiones sociales se correlaciona significativamente con el nivel de felicidad. En otro estudio, también longitudinal, en Alemania, se encontró que el incremento del capital social fue el predictor más importante de la felicidad (Bartolini, Bilancini, \& Sarracino, 2013). El capital social se refiere a los vínculos positivos disponibles para individuos o grupos donde prevalecen las actitudes o sentimientos de ayuda o amabilidad (Kwon \& Adler, 2014). Bartolini y Sarracino (2014) utilizando datos de 27 países (tanto desarrollados como no) distinguieron los efectos del capital social en la felicidad en el corto, mediano y largo plazo. Mientras que en el corto plazo dicho efecto es débil, se fortalece sustantivamente en el mediano y largo plazo.

Otra importante cantidad de estudios se han centrado en la personalidad como predictor de la felicidad. Muchos de ellos hallaron una relación negativa robusta de la felicidad con el neuroticismo y positiva con la extraversión (e.g. Chico-Libran, 2006; Vittersø \& Nilsen, 2002). Andrés et al. (2014) encontraron que la reevaluación cognitiva, es decir la construcción de significados alternativos positivos de un evento, media entre la extraversión y la felicidad. Steel, Schmidt y Shultz (2008) descubrieron que se relacionan de modo positivo con la felicidad la agradabilidad, la escrupulosidad y la apertura a la experiencia.

En América Latina, la felicidad es uno de los constructos más frecuentemente estudiados por la psicología positiva (Castro, 2013). Se destacan los estudios de Alarcón (2006), que concibe a la felicidad como un estado afectivo de satisfacción plena que experimenta subjetivamente el individuo en posesión de un bien anhelado. Encontró que está compuesta por las dimensiones Sentido Positivo de la Vida, Satisfacción con la Vida, Realización Personal y Alegría de Vivir.

En Latinoamérica se han encontrado valores relativamente altos de felicidad, aun considerando los estándares internacionales más demandantes (Calvo et al., 2012). Esto constituye una aparente paradoja dadas sus altas tasas de pobreza, diferencias sociales e inequidad de ingresos y al bajo nivel de acceso a la salud y a la educación en relación a Estados Unidos y Europa Occidental (Beytía, 2016; Rojas, 2016).

Beytía (2016) argumenta que para poder entender los niveles de felicidad relativamente altos de la región es necesario considerar especialmente la calidad de los lazos familiares. El autor distingue entre factores asociados directamente con el nivel de felicidad en Latinoamérica y factores indirectos, que actúan como mediadores de la misma. Los factores directos hallados son la confianza en las relaciones cercanas, el sentimiento de libertad y control, la percepción subjetiva de salud, la satisfacción financiera, el nivel de ingresos, la edad, la religiosidad, el desempleo y 
el hecho de convivir con una pareja. Los factores indirectos fueron el género, el nivel educativo, la confianza institucional y la confianza en las relaciones no cercanas.

Dentro de los factores directos, la satisfacción financiera y el nivel de ingresos tienen menos centralidad en la red de condiciones asociadas con la felicidad en América Latina en comparación con otras regiones. El nivel de confianza institucional no difiere con otras regiones. Pero existe un tipo de relación social, el lazo familiar, en el cual se encuentran diferencias. En América Latina las familias tienen mayor tamaño y están acopladas, generando ayuda mutua, sentimientos de orgullo y vínculos cercanos (Beytía, 2016). Estas características familiares podrían explicar la felicidad de esta población y se observan especialmente en grupos tradicionales. El colectivismo interdependiente propio de Latinoamérica parece estar articulado en una red progresiva, con la familia en el centro, los amigos cercanos en forma contigua, y la comunidad y el vecindario en una capa más exterior (Yamamoto, 2016).

En un estudio cuantitativo realizado en la ciudad de Manizales, Colombia (Velásquez, 2016) se halló que los factores que afectaron el bienestar subjetivo y la felicidad fueron el ingreso del hogar, el sexo y la edad de la persona, así como la satisfacción con diversos dominios vitales como situación económica, situación habitacional, trabajo, salud, familia, vida sexual, relaciones sociales, tiempo libre y logros personales. En un segundo estudio de corte cualitativo se encontró que la experiencia de bienestar es fortalecida por el acceso a diferentes tipos de recursos materiales y afectivos que son considerados valiosos desde la perspectiva del sujeto. Para los habitantes de Manizales, los aspectos más valorados para alcanzar el bienestar fueron la familia, el trabajo, la salud y la educación.

En otro estudio, Caycho, Contreras y Merino (2016) encontraron que en adolescentes y jóvenes de
Lima Metropolitana las dimensiones de la percepción de estilos de crianza correlacionaron en forma significativa con las dimensiones de la Escala de Felicidad de Lima (Alarcón, 2006). Los autores hallaron que el estilo de crianza de rechazo por parte del padre y la calidez emocional de la madre, predicen significativamente la variable felicidad.

La vinculación de la felicidad con el nivel educativo es compleja. En general, bajos niveles de educación formal se relacionan con una salud más descuidada, menor esperanza de vida y menor bienestar (Marmot \& Wilkinson, 2006) así como con actitudes que apuntan contra la cohesión social tales como prejuicio, falta de interés en la política y falta de confianza en otros (Elchardus \& De Keere, 2013).

En numerosos estudios se detectó una relación positiva entre felicidad y nivel educativo. Por ejemplo, Easterbrook, Kuppens y Manstead (2015) estudiaron una muestra de población general inglesa hallando que el nivel de educación es un predictor del bienestar y además se asocia positivamente con el interés político, la confianza social y los niveles de salud y de aptitudes intergrupales. Por su parte, Cuñado y Pérez de Gracia (2011) analizaron datos de población general española identificando efectos positivos tanto directos como indirectos de la educación en la felicidad. Interpretaron el efecto directo como resultado de un aumento en la autoestima y confianza por el hecho de haber adquirido conocimientos, mientras que el efecto indirecto lo consideraron en aquellas personas que con mayor nivel educativo tienden a tener mayor nivel de ingresos y probabilidad de conseguir un mejor trabajo. Por estos efectos a mayor educación se reportarían mayores niveles de felicidad.

Sin embargo, en otros casos no se observa que un mayor nivel educativo se asocie con una mayor felicidad. Ahn y Mochón (2010) no encontraron una relación entre estas dos variables utilizando datos de población adulta española. González-Quiñones y 
Restrepo-Chavarriaga (2010) utilizando una muestra de población general colombiana proveniente de zona urbana (Bogotá, 67\%) y rural (Ubaté, 33\%) tampoco hallaron diferencias entre los varones en el nivel de felicidad en función del nivel educativo. Asimismo, Gerstenbluth, Rossi y Triunfo (2008) no detectaron una relación entre felicidad y nivel educativo en argentinos.

Kit-Chun y Pak-Wai (2013) compararon los determinantes de la felicidad para China y Estados Unidos. Según sus hallazgos, en China los individuos con bajos niveles educativos experimentan una felicidad significativamente menor que aquellos con más educación. En Estados Unidos, en cambio, no se observaron diferencias sistemáticas en felicidad entre individuos provenientes de diferentes niveles educativos.

En la mayoría de los países, las mujeres se autorreportaron con mayores niveles de felicidad (Graham \& Chattopadhyay, 2013). En un estudio realizado con una muestra intencional de sujetos de la ciudad de Buenos Aires con una media de edad de 28 años por Zubieta, Muratori y Fernández (2012) hallaron que los varones puntúan más alto en la capacidad de resistencia a la presión social mientras que las mujeres muestran valores mayores en el interés por el desarrollo personal y en la calidad de las relaciones sociales. Estos resultados pueden estar asociados a las distintas formas de experimentar la felicidad.

En cuanto al efecto del nivel educativo en la felicidad teniendo en cuenta al género, SalinasJiménez, Artés y Salinas-Jiménez (2013) consideraron que la educación tiene un efecto directo, por sí misma, incrementando el bienestar de las mujeres; mientras que para los varones posee una importancia más instrumental. En este último caso, la satisfacción se deriva de la educación a través del aumento en las oportunidades laborales y de status.
En síntesis, las diferencias de género y de nivel educativo, así como su interacción muy posiblemente afecten la felicidad de las personas. Resulta importante, en un marco internacional de progresivo cumplimiento de los Derechos Humanos, poder garantizar equidad entre los individuos lo cual contribuye a su felicidad. Dicha equidad se vería reflejada en una relativa homogeneidad de los niveles de felicidad sin importar si se trata de un varón o una mujer; de un profesional o una persona con un nivel medio de educación. También es importante conocer si el género y el nivel educativo interaccionan para explicar la felicidad y así poder explorar qué grupos sociales son los más vulnerables. Esta es una información de utilidad para diseñar políticas sociales que promuevan la inclusión social.

El objetivo general de este artículo es explorar si existe un efecto del género y el nivel educativo en la felicidad de adultos argentinos con un mínimo de 12 años aproximados de escolaridad. Los objetivos específicos son, en primer lugar, en el caso de existir un efecto significativo, describir qué niveles educativos se asocian con los mayores y menores niveles de felicidad. Además, en el caso de existir un efecto significativo, describir qué género se asocia con los mayores y menores niveles de felicidad. También es un objetivo específico explorar si la interacción entre nivel educativo y género resulta estadísticamente significativa para explicar variaciones en los niveles de felicidad. Por último, dar posibles interpretaciones a los resultados obtenidos, cimentando futuras investigaciones.

\section{Método}

\section{Participantes}

Mediante un diseño muestral por accesibilidad se seleccionaron tres muestras procurando su independencia ya que los instrumentos fueron administrados en diferentes ubicaciones dentro de la misma localidad para cada muestra, cada una de 200 participantes argentinos con igual cantidad de varones 
y mujeres (media de edad de 30.6 años con una desviación estándar de 10.9 años; 19\% de la Ciudad Autónoma de Buenos Aires [CABA], 31\% del Gran Buenos Aires [GBA], 18\% del Interior de la Provincia de Buenos Aires [IPBA], 32\% del Interior de la Argentina[IA],): G1 compuesta por individuos con nivel secundario completo; G2 por individuos con terciario incompleto (universitario y no universitario) y G3 por individuos con nivel terciario completo (universitario y no universitario). Un nivel secundario completo representa 12 años totales aproximados de escolarización, un nivel terciario incompleto entre 13 y 16 años aproximados de escolarización mientras que un nivel terciario completo supone 17 años totales o más aproximados de escolarización. Para disminuir la posible influencia del nivel socioeconómico, no se seleccionaron individuos con primario completo o incompleto y secundario incompleto.

A cada participante se le solicitó el consentimiento informado. Se destacó que la información recabada solo sería utilizada con fines de investigación y que la participación era totalmente voluntaria. Se solicitó sinceridad al responder, explicando que no hay respuestas correctas o incorrectas. La investigación, de esta manera, se ajustó a las pautas éticas propuestas por la American Psychological Association (2010).

En la Tabla 1 se presenta la media y desviación estándar de edad de mujeres y varones para cada nivel educativo y región. Se observa una relativa homogeneidad de las medias y desviaciones

Tabla 1

Media y desviación estándar de edad de mujeres y varones según Nivel Educativo y Región

\begin{tabular}{|c|c|c|c|}
\hline & \multicolumn{3}{|c|}{ Nivel educativo } \\
\hline & G1 & G2 & G3 \\
\hline & \multicolumn{3}{|c|}{ Mujeres $(n=300)$} \\
\hline Edad Media & $26.5(9.2)$ & $27.9(9.6)$ & $37.6(11.7)$ \\
\hline \multicolumn{4}{|l|}{ Región } \\
\hline CABA & 29.2(12.5) & 28.7(10.0) & $38.1(14.5)$ \\
\hline GBA & $28.5(9.1)$ & 29.9(10.8) & $36.7(10.9)$ \\
\hline IPBA & $25.1(7.4)$ & 28.7(12.2) & $39.5(12.2)$ \\
\hline \multirow[t]{2}{*}{ IA } & $23.0(6.4)$ & 25.7(6.9) & $37.1(11.0)$ \\
\hline & \multicolumn{3}{|c|}{ Varones $(n=300)$} \\
\hline Edad Media & $27.9(10.5)$ & $27.9(9.1)$ & $34.8(10.3)$ \\
\hline \multicolumn{4}{|l|}{ Región } \\
\hline CABA & $30.7(10.9)$ & $27.4(10.5)$ & $38.5(11.2)$ \\
\hline GBA & 29.8(12.3) & $28.6(8.7)$ & $34.5(9.5)$ \\
\hline IPBA & $23.5(5.5)$ & $34.5(11.7)$ & $33.0(6.6)$ \\
\hline IA & $25.5(8.9)$ & $25.4(6.7)$ & $32.8(10.7)$ \\
\hline
\end{tabular}

Nota: G1: Máximo nivel de educación alcanzado Secundario completo, G2: Máximo nivel de educación alcanzado Terciario incompleto (Universitario y No Universitario), G3: Máximo nivel de educación alcanzado Terciario completo (Universitario y No Universitario), CABA: Zona de Residencia Ciudad Autónoma de Buenos Aires, GBA: Zona de Residencia Gran Buenos Aires, IPBA: Zona de Residencia Interior de la Provincia de Buenos Aires, IA: Zona de Residencia Interior de la Argentina. 
estándar de edad entre mujeres y varones según nivel educativo como así también de las medias por región entre G1 y G2. En cuanto a G3 naturalmente tiene una media de edad superior a las anteriores por ser el mayor nivel de educación, Terciario completo (Universitario y No Universitario), mostrando una relativa homogeneidad por región.

\section{Instrumento}

Se administró un cuestionario sociodemográfico que incluía una pregunta acerca del máximo nivel educativo alcanzado, que se denominará de ahora en adelante «nivel educativo».

Para evaluar la felicidad se aplicó la versión argentina de la Escala de Felicidad de Lima (EFL) de Alarcón (2006). La EFL ha sido adaptada en varios países latinoamericanos: México (Toribio, González, Valdez, González, \& Oudhof, 2012), Venezuela (Arraga \& Sánchez, 2010, 2012), Brasil (Medeiros, Nascimento, Mariano, Sales, \& Medeiros, 2014) y Argentina (Auné, Abal, \& Attorresi, 2017).

En la adaptación argentina de dicho instrumento se eliminaron las dimensiones Realización Personal y Alegría de Vivir y se desdobló Sentido Positivo de la Vida. Dado que en el trabajo original de Alarcón (2006) se obtuvo un modelo de cuatro factores se impuso en primer lugar en el estudio de adaptación una estructura tetrafactorial. Sin embargo, la dimensionalidad de la EF adaptada resultó inconsistente con la estructura teórica propuesta para la prueba original ya que en uno de los factores originales cargaron sólo dos ítems, los cuales no son suficientes para constituir una dimensión (Velicer \& Fava, 1998). Dado que no se pudo sostener la estructura tetrafactorial con los 27 ítems originales, se realizó una depuración de los reactivos que cumplían con criterios de eliminación. Fueron excluidos de los análisis posteriores los ítems 5 ( $L a$ vida ha sido buena conmigo), 6 (Me siento satisfecho con lo que soy), 11 (La mayoría del tiempo no me siento feliz) y 13 (Por lo general, me siento bien) por presentar doble pesaje, es decir que cargaron en dos dimensiones. Asimismo, fueron eliminados los ítems 1 (En general, mi vida está cerca de mi ideal), 8 (Hasta ahora he conseguido las cosas que para mí son importantes), 9 (Si volviera a nacer no cambiaría casi nada de mi vida), 12 (Es maravilloso vivir), 15 (Soy una persona optimista), 16 (He experimentado la alegría de vivir), 21 (Estoy satisfecho con lo que hasta ahora he alcanzado), 24 (Me considero una persona realizada), 25 (Mi vida transcurre plácidamente) y 27 (Creo que no me falta nada) por cargar en un factor que no les correspondía según los hallazgos de Alarcón (2006). Por último, se eliminó el ítem 14 (Me siento inútil) por presentar correlaciones insuficientes (menores a .30) en todos los factores, es decir en los cuatro factores originales. Posteriormente, se realizó un nuevo AFE imponiendo una estructura trifactorial. Los datos fueron adecuados para realizar dicho procedimiento (KMO $=$.88; prueba de esfericidad de Bartlett, $\chi 2=4984.3$, $\mathrm{gl}=66, p<.001)$. El nivel de varianza explicada se considera alto (69\%), sugiriendo que los factores representaban un elevado porcentaje de la variación del conjunto de datos. El Análisis Paralelo, a su vez, indicó que el número de factores extraído era adecuado. Los tres factores hallados implicaron un desdoblamiento de la dimensión Sentido Positivo de la Vida.

Es una adaptación de doce ítems y con cinco opciones de respuesta de tipo Likert que se puntúan del uno al cinco tal como lo propuso Alarcón (2006) $(1=$ Totalmente en desacuerdo, 2 = Desacuerdo, 3 = Ni acuerdo ni desacuerdo; 4 = Acuerdo; 5 = Totalmente de acuerdo).

El instrumento cuenta con evidencias de validez factorial y estudios de confiabilidad suficientes para su aplicación en la población de estudio. Mide a la felicidad como constructo psicológico a través de tres dimensiones moderadamente correlacionadas que explicaron el 69\% de la varianza: a) Sentido de la Vida Perspectiva Externa (SVPE, cuatro ítems, $\alpha=$ 
.84, en este estudio $\alpha=.78$ ), que se relaciona con la tendencia a experimentar sentimientos positivos hacia el entorno; b) Sentido de la Vida Perspectiva Interna (SVPI, cinco ítems, $\alpha=.89$, en este estudio $\alpha=.80$ ), vinculada con los sentimientos positivos hacia sí mismo y c) Satisfacción con la Vida (SCV, tres ítems, $\alpha=.75$, en este estudio $\alpha=.76$ ) que expresa satisfacción por lo que se ha alcanzado. Es decir, fue importante para la muestra de adultos argentinos distinguir al exterior o al propio yo como causas de felicidad. Se aportaron evidencias de validez convergente a través de la correlación positiva y significativa con los puntajes en la Escala de Gratitud de Alarcón (2014) adaptación argentina realizada por Auné, Abal y Attorresi (2017) y en la Escala de Conducta Prosocial (ECP; Auné, Abal, \& Attorresi, 2016b).

\section{Procedimiento}

La presente investigación constituye un estudio transversal, de campo, en formato de encuesta descriptiva. No pertenece a la categoría experimental ya que ninguna de las variables independientes fue manipulada (Montero \& León, 2005). La selección de los participantes fue por accesibilidad mediante la técnica de muestreo bola de nieve hasta alcanzar la cantidad de datos requeridos con las características especificadas.

\section{Análisis de datos}

Una vez volcados los datos a una base, se aplicó Análisis de la Varianza (ANOVA) de dos vías por separado para cada una de las tres dimensiones de la Felicidad (SVPE, SVPI y SCV) empleando el programa Statistical Package for Social Sciences (SPSS) versión 18. Se estimó el tamaño del efecto mediante el $\eta^{2}$ parcial, que resulta análogo a la proporción de la varianza explicada (Coe \& Merino, 2003). Para el contraste post-hoc se utilizó la prueba de Scheffé ya que este último es un procedimiento más robusto cuando no se cumple el supuesto de normalidad del ANOVA (Hilton \& Armstrong, 2006).
El puntaje total de cada dimensión se adoptó como variable dependiente, mientras que género (varón o mujer) y nivel educativo (G1, G2 o G3) se configuraron como factores fijos. Se incluyó en género la categoría otro, pero al tener sólo un participante que eligió esta opción no se utilizó para el análisis estadístico. No se incluyó la edad como covariable en el ANOVA dado que se considera que el tamaño de su efecto es nulo para las tres dimensiones (correlación de Pearson, SVPE, $r=$ .079; SVPI, $r=.084$; SCV, $r=-.001$ ) según el criterio de Manzano (2009).

El contraste de los supuestos de homocedasticidad con la prueba de Levene y de normalidad con la prueba de Kolmogorov-Smirnov indicó que para las tres dimensiones no se rechaza la homocedasticidad pero sí la normalidad (SVPE, prueba de Levene, $p=.49$, prueba de KolmogorovSmirnov, $p<.001$; SVPI, prueba de Levene, $p=.33$, prueba de Kolmogorov-Smirnov, $p<.001$; SCV, prueba de Levene, $p=.81$; prueba de KolmogorovSmirnov, $p<.001$ ). Es de destacar que el ANOVA es robusto al incumplimiento del supuesto de normalidad mientras que se cumpla la homocedasticidad (Pardo \& San Martin, 1994). Además, tomar muestras de igual tamaño permite que, si existen violaciones a los supuestos de homocedasticidad y normalidad, las mismas tengan considerablemente menos efecto (Aron, Coups, \& Aron, 2011; Lomax \& Hahs-Vaughn, 2012).

Para la comparar los coeficientes de confiabilidad alfa de Cronbach por nivel educativo se implementó el programa AlphaTest (Lautenschlager \& Meade, 2008) el cual permite incluir los alfas de más de dos subgrupos. Para la comparación de coeficientes de confiabilidad por género se utilizó asimismo el programa AlphaTest y además se efectuó mediante MS Excel el procedimiento de Feldt y Kim (2006) para comparar entre alfas cuando el tamaño del test o el tamaño de la muestra es pequeño. 


\section{Resultados}

Se presentarán análisis descriptivos e inferenciales. El análisis descriptivo se expone en la Tabla 2 donde pueden apreciarse las medias y desviaciones estándar para cada dimensión según género y nivel educativo.

Los análisis inferenciales fueron los resultados del ANOVA para las dimensiones SVPE, SVPI y SCV, los cuales se exponen en la Tabla 3.
Los resultados del ANOVA para la dimensión SVPE indican que nivel educativo y la interacción género- nivel educativo tienen efectos significativos sobre la media de puntaje en SVPE) con tamaños del efecto pequeños. En cambio, el efecto simple de género sobre la media de puntaje en SVPE no fue estadísticamente significativo. Las comparaciones post-hoc para nivel educativo mediante la prueba de Scheffé señalan que el grupo G1 obtiene puntuaciones significativamente menores que el grupo G3 $(p=.027)$.

\section{Tabla 2}

Media y desviación estándar de puntaje por dimensión de la EFL según Género y Nivel Educativo

\begin{tabular}{|c|c|c|c|}
\hline & \multicolumn{3}{|c|}{ Nivel educativo } \\
\hline & G1 & G2 & G3 \\
\hline & \multicolumn{3}{|c|}{ Mujeres $(n=300)$} \\
\hline \multicolumn{4}{|c|}{ Dimensión } \\
\hline SVPE & $15.18(3.56)$ & $16.37(3.28)$ & $16.40(3.25)$ \\
\hline SVPI & 18.11(4.58) & $19.37(4.17)$ & $19.91(4.44)$ \\
\hline \multirow[t]{2}{*}{ SCV } & $9.77(2.54)$ & $10.62(2.56)$ & $10.40(2.51)$ \\
\hline & \multicolumn{3}{|c|}{ Varones $(n=300)$} \\
\hline \multicolumn{4}{|c|}{ Dimensión } \\
\hline SVPE & $16.05(3.40)$ & $15.40(3.26)$ & $16.34(2.83)$ \\
\hline SVPI & $19.15(4.61)$ & $18.60(3.86)$ & $19.76(3.72)$ \\
\hline SCV & $9.99(2.77)$ & $9.53(2.65)$ & $10.18(2.37)$ \\
\hline
\end{tabular}

Nota: G1: Máximo nivel de educación alcanzado Secundario completo, G2: Máximo nivel de educación alcanzado Terciario incompleto (Universitario y No Universitario), G3: Máximo nivel de educación alcanzado Terciario completo (Universitario y No Universitario), SVPE: Dimensión de la Escala de Felicidad de Lima Sentido de la Vida Perspectiva Externa, SVPI: Dimensión de la Escala de Felicidad de Lima Sentido de la Vida Perspectiva Interna, SCV: Dimensión de la Escala de Felicidad de Lima Satisfacción con la Vida. 
Tabla 3

Resultados obtenidos en el ANOVA de dos factores en relación las Dimensiones SVPE, SVPI y SCV

\begin{tabular}{lcccc}
\hline & F & gl & $p$ & $\eta^{2}$ parcial \\
\hline Dimensión & & & & \\
\hline SVPE & & & & \\
G & .13 & 1 & .64 & .000 \\
NE & 4.12 & 2 & .02 & .014 \\
G*NE & 4.14 & 2 & .02 & .014 \\
SVPI & & & & \\
G & .01 & 1 & .91 & .000 \\
NEG & 4.24 & 2 & .02 & .014 \\
$*$ NE & 2.34 & 2 & .09 & .008 \\
SCV & & & & \\
G & 3.03 & 1 & .08 & .005 \\
NE & 1.26 & 2 & .28 & .004 \\
G*NE & 3.34 & 2 & .04 & .011
\end{tabular}

Nota: G: Género, NE: Nivel Educativo, G*NE: Interacción Género-Nivel Educativo, SVPE: Dimensión de la Escala de Felicidad de Lima Sentido de la Vida Perspectiva Externa, SVPI: Dimensión de la Escala de Felicidad de Lima Sentido de la Vida Perspectiva Interna, SCV: Dimensión de la Escala de Felicidad de Lima Satisfacción con la Vida.

En la Figura 1 se puede apreciar cómo las medias del puntaje total en la dimensión SVPE aumentan levemente de G1 a G2 y lo hacen de forma más pronunciada en G3.

En el Figura 2 se comparan las medias del puntaje total en la dimensión SVPE según género y nivel educativo.

Los resultados del ANOVA para SVPI indican que nivel educativo y la interacción entre género y nivel educativo si se considera $\alpha=.10$ tienen efectos significativos sobre la media de puntaje en SVPI con tamaños del efecto pequeños. En cambio, el efecto simple del género sobre la media de puntaje en SVPI no fue estadísticamente significativo. Las comparaciones post-hoc para nivel educativo mediante la prueba de Scheffé señalan que el grupo G1 obtiene puntuaciones significativamente menores que el grupo G3 $(p=.019)$.

En la Figura 3 se puede apreciar cómo las medias del puntaje total en la dimensión SVPE aumentan levemente de G1 a G2 y lo hacen de forma más pronunciada en G3. 


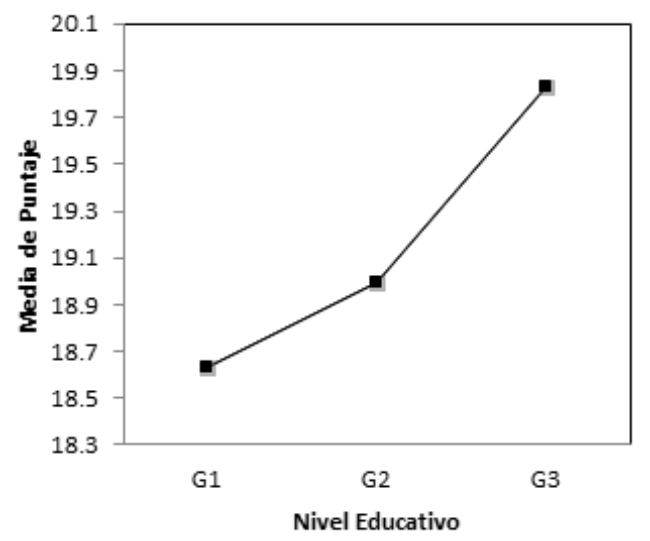

Figura 1. Medias del puntaje por nivel educativo en la dimensión SVPE Nivel Educativo

G1: Secundario incompleto; G2: Terciario incompleto (Universitario y No Universitario); G3: Terciario completo (Universitario y no Universitario).

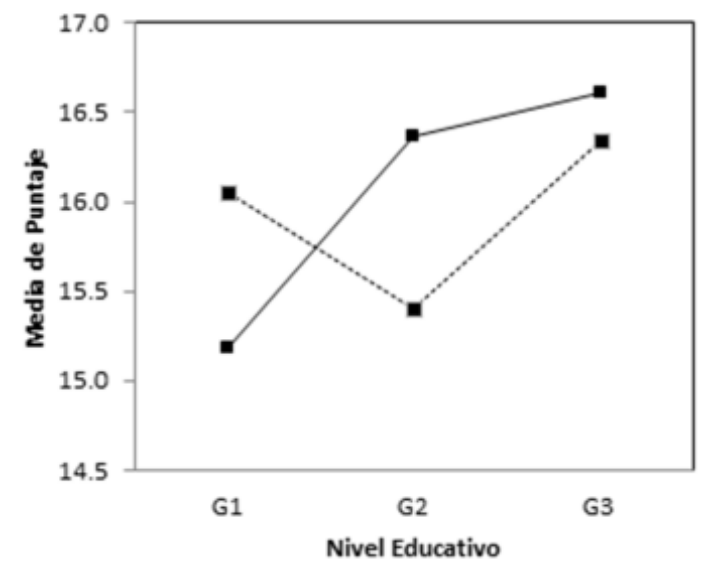

Figura 2. Medias del puntaje por nivel educativo según género en la dimensión SVPE Género Mujer - Varón ---

Nivel Educativo

G1: Secundario incompleto; G2: Terciario incompleto (Universitario y No Universitario); G3: Terciario completo (Universitario y no Universitario). 


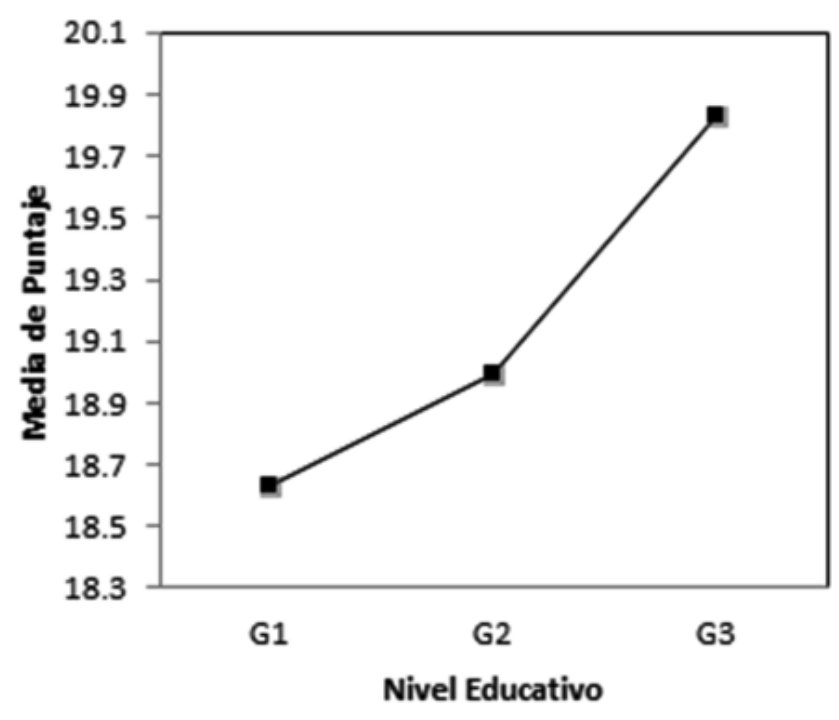

Figura 3. Medias del puntaje por nivel educativo en la dimensión SVPI Nivel Educativo G1: Secundario incompleto; G2: Terciario incompleto (Universitario y No Universitario); G3: Terciario completo (Universitario y no Universitario).

En la Figura 4 se comparan las medias del puntaje total en la dimensión SVPI según género y nivel educativo.

Los resultados del ANOVA para SCV indican que el efecto simple de género si se considera $\alpha=.10 \mathrm{y}$ la interacción género- nivel educativo tienen efectos significativos sobre la media de puntaje en SCV con tamaños del efecto pequeños. Las medias de puntaje por género fueron de 10.3 (2.5) para las mujeres y 9.9 (2.6) para los varones. En cambio, el efecto simple de nivel educativo sobre la media de puntaje en SCV no fue estadísticamente significativo.
En la Figura 5 se comparan las medias del puntaje total en la dimensión SCV según género y nivel educativo.

Se calculó además el Alfa de Cronbach por separado para cada género y nivel educativo, como se puede observar en las Tablas 4 y 5 . Los estadísticos correspondientes a la comparación entre alfas también se incluyen en dichas Tablas. Sólo en la comparación de alfas por nivel educativo para la dimensión SPVE se rechaza la hipótesis nula de igualdad entre ellos. 


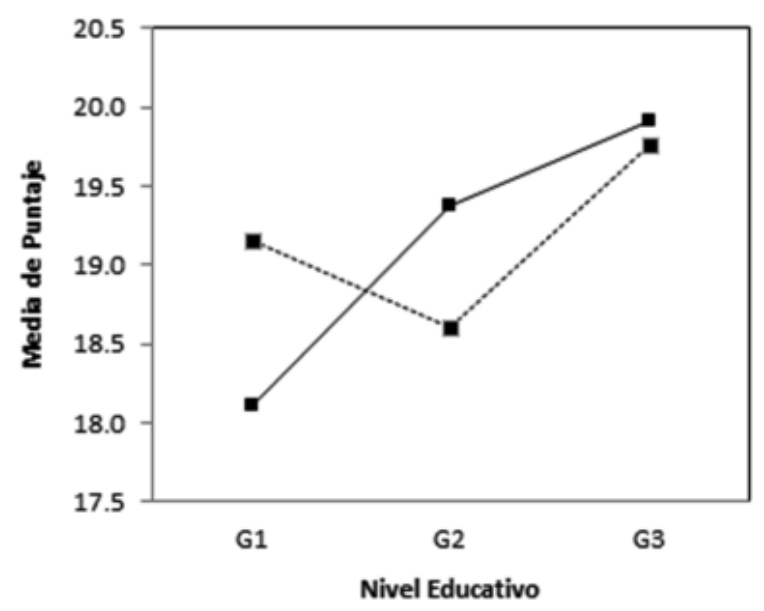

Figura 4. Medias del puntaje por nivel educativo según género en la dimensión SVPI Género Mujer - Varón ---

Nivel Educativo

G1: Secundario incompleto; G2: Terciario incompleto (Universitario y No Universitario); G3: Terciario completo (Universitario y no Universitario).

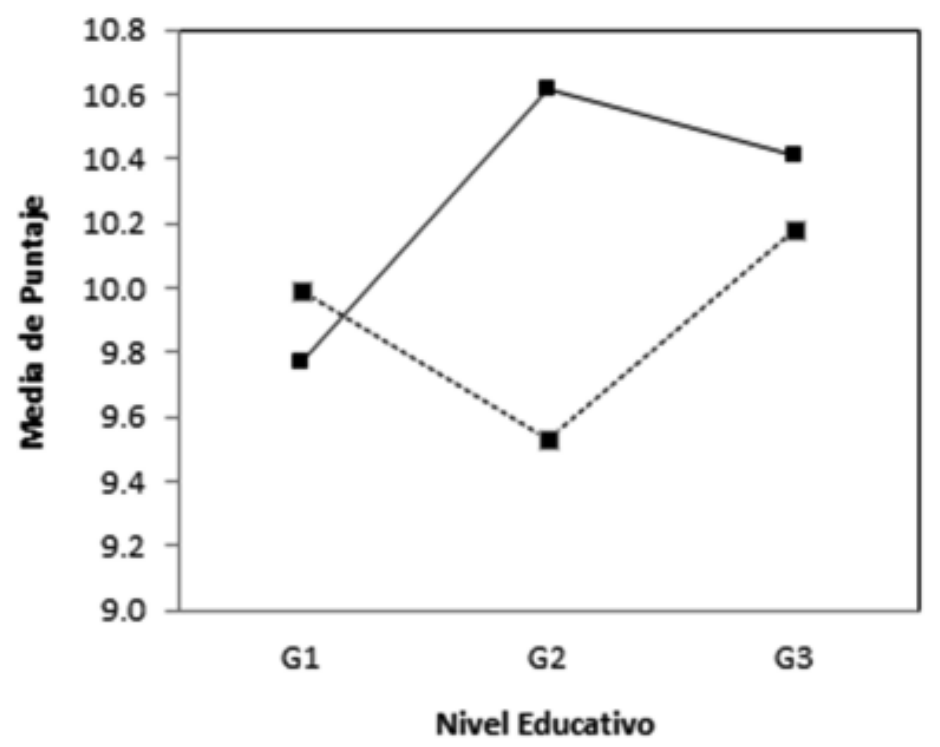

Figura 5. Medias del puntaje por nivel educativo según género en la dimensión SCV Género

Mujer - Varón ---

Nivel Educativo

G1: Secundario incompleto; G2: Terciario incompleto (Universitario y No Universitario); G3: Terciario completo (Universitario y no Universitario). 
Tabla 4

Alfa de Cronbach para cada Dimensión en función del Género y Pruebas de Diferencia

\begin{tabular}{lccccccccc}
\hline & $\mathrm{M}$ & $\mathrm{V}$ & \multicolumn{1}{c}{$\chi^{2}$} & $\mathrm{gl}$ & \multicolumn{1}{c}{$p$} & $\mathrm{~W}$ & $\mathrm{gl}_{\mathrm{M}}$ & $\mathrm{gl}_{\mathrm{v}}$ & $p$ \\
\hline SVPE & .79 & .77 & .37 & 1 & .54 & 1.09 & 224 & 224 & .25 \\
SVPI & .81 & .78 & 1.07 & 1 & .30 & .86 & 239 & 239 & .87 \\
SCV & .74 & .77 & .56 & 1 & .45 & 1.13 & 199 & 199 & .19 \\
\hline
\end{tabular}

Nota: M: Mujeres, V: Varones, SVPE: Dimensión de la Escala de Felicidad de Lima Sentido de la Vida Perspectiva Externa, SVPI: Dimensión de la Escala de Felicidad de Lima Sentido de la Vida Perspectiva Interna, SCV: Dimensión de la Escala de Felicidad de Lima Satisfacción con la Vida. W $=1-\alpha_{1} / 1-\alpha_{2}$. W sigue la distribución $\mathrm{F}$ con grados de libertad $\mathrm{gl}_{\mathrm{M}}$ (grados de libertad del subgrupo Mujeres) y gl (grados de libertad del subgrupo Varones).

\section{Tabla 5}

Alfa de Cronbach para cada Dimensión en función del Nivel Educativo y Pruebas de Diferencia

\begin{tabular}{llllrll}
\hline & G1 & G2 & G3 & \multicolumn{1}{c}{$\chi^{2}$} & gl & \multicolumn{1}{c}{$p$} \\
\hline SVPE & .83 & .78 & .69 & 10.56 & 2 & $.005^{*}$ \\
SVPI & .81 & .76 & .82 & 3.59 & 2 & .17 \\
SCV & .77 & .74 & .75 & .39 & 2 & .82 \\
\hline
\end{tabular}

Nota: G1: Máximo nivel de educación alcanzado Secundario completo, G2: Máximo nivel de educación alcanzado Terciario incompleto (Universitario y No Universitario), G3: Máximo nivel de educación alcanzado Terciario completo (Universitario y No Universitario), SVPE: Dimensión de la Escala de Felicidad de Lima Sentido de la Vida Perspectiva Externa, SVPI: Dimensión de la Escala de Felicidad de Lima Sentido de la Vida Perspectiva Interna, SCV: Dimensión de la Escala de Felicidad de Lima Satisfacción con la Vida.

${ }^{*} p<.01$ 


\section{Discusión}

Los resultados indicarían que el nivel educativo establece diferencias en dos de las dimensiones de la EFL. Esto es coherente con la relación positiva entre felicidad y nivel educativo hallada por Easterbrook et al. (2015) y Cuñado y Pérez de Gracia (2011) entre otros estudios. Sin embargo, la magnitud del tamaño del efecto es muy pequeña indicando que un porcentaje mínimo de la felicidad es explicado por el nivel educativo. Podría tratarse, como lo señala Beytía (2016) de un factor mediador de la felicidad.

En SVPE y SVPI se observa una diferencia de puntaje a favor de los grupos de mayor nivel educativo. La primera de estas dimensiones se relaciona con el exterior como causa de la felicidad o infelicidad y podría estar vinculada al locus de control externo. La dimensión SVPI describe la capacidad de sentir felicidad y atribuirla al propio yo. Ambos resultados pueden estar reflejando la influencia del nivel económico (Gerstenbluth et al., 2008) y de la calidad de vida en general. Esto es una posible hipótesis ya que no se cuenta con información recogida en el presente estudio acerca de estas variables. Además, Oreopoulos y Salvanes (2011) señalaron que los trabajadores con mayor nivel educativo disfrutarían de más beneficios no monetarios del empleo, incluyendo una mayor sensación de logro, prestigio profesional y una mayor satisfacción laboral.

No se hallaron diferencias a favor de los grupos de mayor nivel educativo en la dimensión SCV. La misma se relaciona con lo que se ha alcanzado, la distancia con un ideal. La educación incrementa el sentimiento de logro pero también las expectativas, que el caso de no consumarse tal vez generarían esta falta de diferencias significativas entre los grupos. Es así que en las personas con mayor nivel educativo se produciría también una distancia importante entre la satisfacción por lo que han alcanzado y las metas a futuro, disminuyendo así su nivel de satisfacción actual (Ahn \& Mochón, 2010).
Asimismo, es importante reconocer la influencia de la deseabilidad social, la cual a pesar de ser anónima la administración de la escala, podría estar influyendo sobre las tres dimensiones. Es difícil reconocerse ante sí mismo como infeliz (Veenhoven, 1998) y este hecho puede atenuar las diferencias en puntaje entre los individuos con distinto grado de educación formal.

La interacción entre el género y el nivel educativo fue estadísticamente significativa para explicar el puntaje en las tres dimensiones de la EFL. Sin embargo, la magnitud del tamaño del efecto es muy pequeña indicando que un porcentaje mínimo de la felicidad es explicado por esta interacción. Las mujeres con menor nivel educativo (secundaria completa) son el único grupo con puntaje menor al de los varones en las tres dimensiones. La mujer con bajo nivel educativo puede sentir más vulnerabilidad y dependencia económica y social que el varón con igual nivel, en el sentido, por ejemplo, de participar escasamente en ámbitos públicos con la consecuente dificultad para la obtención de ingresos (Miranda, 2008). En este grupo es más probable que haya un porcentaje más alto de amas de casa sin independencia económica que en los otros niveles educativos. Esto es una posible hipótesis ya que no se cuenta con información recogida en el presente estudio acerca del porcentaje de amas de casa. A pesar de la mejora de las condiciones de la mujer en el mundo social y del trabajo, todavía quedan remanentes de conductas de una época con fuerte predominio masculino y una tendencia en los mercados de trabajo a un desbalance por género (Elborgh-Woytek et al., 2013).

El hecho de tener un mayor nivel educativo (como mínimo terciario incompleto, sea o no universitario) podría influir para que la media de las mujeres aumente en mayor medida que la de sus pares masculinos. El acceso masivo de la mujer a la vida profesional es relativamente reciente. Contar con educación técnica o profesional puede hacer que estas mujeres se consideren más capaces, así como 
posibilita condiciones ambientales de prestigio y poder más equitativas. Este mayor nivel educativo les permitiría acceder a una autonomía personal, profesional y económica. Es posible que por estos motivos tiendan a manifestar un mayor grado de felicidad. Coincidentemente, Salinas-Jiménez et al. (2013) encontraron que los efectos de la educación en la satisfacción con la vida son levemente más altos para las mujeres. Esto podría deberse a que, para las mujeres, la educación tiene un efecto directo, por sí misma, en la satisfacción con la vida mientras que para los varones presenta una utilidad más instrumental, al aumentar las oportunidades laborales y de status (Salinas-Jiménez et al., 2013).

Los varones con nivel terciario incompleto son los que presentan la mayor brecha con las mujeres, siendo menor su nivel de felicidad. El terciario incompleto implica que no se han finalizado los estudios superiores, y podría existir una expectativa social de que el varón debe trabajar tempranamente a pesar de estar estudiando. Además, en este nivel educativo aún no hay tantos beneficios laborales y de prestigio, por estar el nivel terciario, justamente, incompleto. En este sentido, Abal, Lozzia, Aguerri, Galibert y Attorresi (2007) hallaron en una muestra de estudiantes de Psicología que los varones que no trabajan presentan una menor perseverancia y responsabilidad en la realización de sus tareas que los varones que trabajan, mientras que en las mujeres no hay diferencia significativa.

La felicidad es un fenómeno complejo y multidimensional con un alto grado de subjetividad. Una vez que las necesidades básicas se encuentran satisfechas cobran más importancia las relaciones sociales cercanas y el nivel de salud (Argyle, 2003). También la cultura influye en la percepción de la felicidad. A pesar de ello es frecuente en la bibliografía suponer la universalidad de los procesos psicológicos (Casullo \& Fernández, 2007) caracterizando erróneamente a la felicidad de la misma forma para distintas culturas. Por otra parte, el hecho de vivir en un medio urbano o rural podría interactuar con el nivel educativo influyendo en la felicidad ya que implica actividades económicas, formas de vida y de relacionarse diferenciadas (Santamaría, 2014). Este es otro punto a explorar en posteriores investigaciones.

Si bien este estudio contó con residentes de distintas zonas del país, la muestra es no probabilística, $\mathrm{y}$, por lo tanto, los resultados son puntuales y no generalizables. Otra limitación es que no se cubrieron los diferentes rangos etarios con una cantidad homogénea de participantes. Una limitación importante del presente estudio es la ausencia de un análisis de invarianza de medición entre los subgrupos analizados. Se debe realizar especialmente para la dimensión SVPE y por nivel educativo, donde para los Alfa de Cronbach resultó estadísticamente significativa su diferencia, lo cual podría estar indicando un posible sesgo (Merino \& Lautenschlager, 2003). En futuras investigaciones se podría explorar las relaciones evaluadas en una muestra cuyos participantes tengan una media más alta de edad. Además, sería de utilidad relevar el tipo de ocupación de los participantes e indicadores de nivel económico, nivel de independencia económica de varones y mujeres y de calidad de vida. 


\section{Referencias}

Abal, F., Lozzia, G., Aguerri, M. E., Galibert, M. S., \& Attorresi, H. F. (2007). Construcción de una escala de voluntad de trabajo. Investigaciones en Psicología, 12(1), 7-16.

Ahn, N., \& Mochón, F. (2010). La felicidad de los españoles: factores explicativos. Revista de economía aplicada, 18(54), 5-31.

Alarcón, R. (2006). Desarrollo de una escala factorial para medir la felicidad. Revista Interamericana de Psicología, 40(1), 95-102.

Alarcón, R. (2014). Construcción y valores psicométricos de una escala para medir la gratitud. Acta de Investigación Psicológica, 4(2), 1520-1534. doi: 10.1016/S2007-4719(14)70391-6

American Psychological Association. (2010). Ethical principles for psychologists and code of conduct. Washington, Estados Unidos de América: APA.

Andrés, M. L., Castañeiras, C. E., \& Richaud de Minzi, M. C. (2014). Relaciones entre la personalidad y el bienestar emocional en niños. El rol de la regulación emocional. Cuadernos de Neuropsicología / Panamerican Journal of Neuropsychology, 8(2), 217241. doi: $10.7714 / \mathrm{cnps} / 8.2 .205$

Argyle, M. (2003). The psychology of happiness (2nd ed.). San Petersburgo, Rusia: Piter.

Arraga, M. V., \& Sánchez, M. (2010). Bienestar subjetivo en adultos mayores venezolanos. Revista Interamericana de Psicología, 44(1), 12-18.

Arraga, M. V., \& Sánchez, M. (2012). Validez y confiabilidad de la Escala de Felicidad de Lima en adultos mayores venezolanos. Universitas Psychologica, 11(2), 381-393.

Aron, A., Coups, E., \& Aron, E. (2011). Statistics for psychology - International edition ( $6^{\text {th }}$ ed.). Melbourne, Australia: Pearson Education.

Auné, S. E., Abal, F. J. P., \& Attorresi, H. F. (en prensa). Versión Argentina de la Escala de Felicidad de Lima. Revista Diversitas.

Auné, S. E., Blum, G. D., Abal, F. J. P., Lozzia, G., \& Attorresi, H. F. (2014). La conducta prosocial: estado actual de la investigación. Perspectivas en Psicología, 11(2), 2133.
Auné, S. E., Abal, F. J. P., \& Attorresi, H. F. (2016a). Antagonismos entre concepciones de empatía y su relación con la conducta prosocial. Revista de Psicología, 17(2), 137-149. doi: 10.18050/ revpsi.v17n2a7.2015

Auné, S., Abal, F., \& Attorresi, H. F. (2016b). Diseño y construcción de una escala de conducta prosocial para adultos. Revista Iberoamericana de Diagnóstico y Evaluación Psicológica, 42(2), 15-25. doi: 10.21865/ RIDEP42_15

Auné, S. E., Abal, F. J. P., \& Attorresi, H. F. (2017). Versión Argentina de la Escala de Gratitud de Alarcón. Manuscrito enviado para su publicación.

Bartolini, S., \& Sarracino, F. (2014). Happy for how long? How social capital and economic growth relate to happiness over time. Ecological Economics, 108, 242256. doi: 10.1016/j.ecolecon.2014.10.004

Bartolini, S., Bilancini, E., \& Pugno, M. (2013). Did the decline in social connections depress Americans' happiness? Social Indicators Research, 110(3), 10331059. doi: 10.1007/s11205-011-9971-X

Bartolini, S., Bilancini, E., \& Sarracino, F. (2013). Predicting the trend of well-being in Germany: How much do comparisons, adaptation and sociability matter? Social Indicators Research, 114(2), 169-191. doi: 10.1007/ s11205-012-0142-5

Beytía, P. (2016). The singularity of Latin American patterns of happiness. En Mariano Rojas (Ed.), Handbook of Happiness Research in Latin America (pp. 17-29). Dordrecht, Holanda: Springer. doi: 10.1007/978-94-0177203-7

Calvo, E., Castellanos, R., Martínez, I., Millán, R., Moyano, E., Palomar, J., ... Yamamoto, J. (2012). Medición, Investigación e Incorporación a la Política Pública del Bienestar Subjetivo: América Latina. México: Foro Consultivo Científico y Tecnológico.

Castro, A. (2013). La psicología positiva en América Latina. Desarrollos y perspectivas. Psiencia. Revista Latinoamericana de Ciencia Psicológica, 4(2), 108116. doi: 10.5872/psiencia.v4i2.95

Casullo, M. M., \& Fernández, M. (2007). Las propuestas de la Psicología Positiva: ¿Universales psicológicos o particulares de una visión cultural? Anuario de 
Investigaciones - Facultad de Psicología de la Universidad de Buenos Aires, 14(1), 261-268.

Caycho, T., Contreras, K., \& Merino, C. (2016). Percepción de los estilos de crianza y felicidad en adolescentes y jóvenes de Lima Metropolitana. Perspectiva de Familia, 1, 11-22

Chico-Libran, E. (2006). Personality dimensions and subjective well-being. Spanish Journal of Psychology, 9, 38-44. doi: 10.1017/S1138741600005953

Coe, R., \& Merino, C. (2003). Magnitud del efecto: Una guía para investigadores y usuarios. Revista de Psicología- PUCP, 21(1), 145-177.

Cuñado, J., \& Pérez de Gracia, F. P. (2011). Does education affect happiness? Evidence for Spain. Social Indicators Research, 108, 185-196. doi:10.1007/s11205-011-9874-X

Diener, E., Sandvik, E., \& Pavot, W. (2009). Happiness is the frequency, not the intensity, of positive versus negative affect. Assessing Well Being, 39, 213-231. doi: 10.1007/978-90-481-2354-4_10

Dzhidaryan, I. A. (2013). Psychology of happiness and optimism. Moscú, Rusia: Publishing House of the «Institute of Psychology, Russian Academy of Sciences».

Easterbrook, M. J., Kuppens, T., \& Manstead, A. S. (2015). The education effect: Higher educational qualifications are robustly associated with beneficial personal and socio-political outcomes. Social Indicators Research, 126(3), 1261-1298. doi: 10.1007/s11205-015-0946-1

Elborgh-Woytek, K., Newiak, M., Kochhar, K., Fabrizio, S., Kpodar, K. Wingender, P., Clements, B., \& Schwartz, G. (2013). Las mujeres, el trabajo y la economía: Beneficios macroeconómicos de la equidad de género. Recuperado de http://www.igualdadenlaempre sa.es/enlaces/webgrafia/docs/Las_mujeres_el_tra bajo_y-FMI.pdf

Elchardus M., \& De Keere K. (2013). Social control and institutional trust: Reconsidering the effect of modernity on social malaise. The Social Science Journal, 50, 101-111. doi: 10.1016/j.soscij.2012.10.004

Feldt, L. S., \& Kim, S. (2006). Testing the difference between two alpha coefficients with small samples of subjects and raters. Educational and Psychological
Measurement, 66(4), 589-600. doi: 10.1177/0013164 405282488

Gerstenbluth, M., Rossi, M., \& Triunfo, P. (2008). Felicidad y salud: una aproximación al bienestar en el Río de la Plata. Estudios de Economía, 35(1), 65-78. doi: 10.4067/ S0718-52862008000100004

González-Quiñones, J. C., \& Restrepo-Chavarriaga, G. (2010). Prevalencia de felicidad en ciclos vitales y relación con redes de apoyo en población colombiana. Revista de Salud Pública, 12(2), 228-238.

Graham, C., \& Chattopadhyay, S. (2013). Gender and wellbeing around the world. International Journal of Happiness and Development, 1(2), 212-232. doi: 10.150 4/IJHD.2013.055648

Grob, A. (1995). Subjective well-being and significant lifeevents across the life span. Swiss Journal of Psychology, 54, 3-18.

Hilton A., \& Armstrong R. (2006). Stat note 6: Post hoc ANOVA tests. Microbiologist, 7, 34-36.

Kit-Chun, L., \& Pak-Wai, L. (2013). Socio-economic inequalities in happiness in China and U.S. Social Indicators Research, 116(2), 509-533.doi: 10.1007/ s11205-013-0283-1

Kwon, S. W., \& Adler, P. S. (2014). Social capital: Maturation of a field of research. Academy of Management Review, 39(4), 412-422. doi: 10.5465/am r.2014.0210

Lautenschlager, G., \& Meade, A. (2008). Alpha Test: A Windows Program for Tests of Hypotheses About Coefficient Alpha. Applied Psychological Measurement, 32(6), 502-503. doi: 10.1177/01466216 07312307

Lomax, R. G., \& Hahs-Vaughn, D. L. (2012). Statistical concepts: A second course. Nueva York, Estados Unidos de América: Routledge.

Lyubomirsky, S., King, L., \& Diener, E. (2005). The benefits of frequent positive affect: Does happiness lead to success? Psychological Bulletin, 131, 803855. doi: 10.1037/0033-2909.131.6.803

Lyubomirsky, S., \& Layous, K. (2013). How do simple positive activities increase well-being? Current Directions in Psychological Science, 22(1), 57-62. doi: $10.1177 / 0963721412469809$ 
Manzano, V. (2009). Sobre la acotación de efectos y la existencia de relación. Recuperado de http:// personal.us.es/vmanzano/docencia/analisis/guias/ efectos.pdf

Marmot, M., \& Wilkinson, R. G. (2006). Social determinants of health. Oxford, Inglaterra: Oxford University Press.

Medeiros, E. D., Nascimento, A. M., Mariano, T. E., Sales, H. F. S., \& Medeiros, P. C. B. (2014). Escala de Felicidade de Lima: Validade fatorial e consistência interna. Psicologia em Pesquisa, 8(2), 150-158. doi: 10.5 327/Z1982-1247201400020004

Miranda, A. (2008). Los jóvenes, la educación secundaria y el empleo a principios del siglo XXI. Revista de Trabajo, 4(6), 185-198.

Merino, C., \& Lautenschlager, G. J. (2003). Comparación estadística de la confiabilidad alfa de Cronbach: aplicaciones en la medición educacional y psicológica. Revista de Psicología, 12(2), 127-136.

Montero, I., \& León, O. G. (2005). Sistema de clasificación del método en los informes de investigación en Psicología. International Journal of Clinical and Health Psychology, 5(1), 115-127.

Oishi, S., Kesebir, S., \& Diener, E. (2011). Income inequality and happiness. Psychological Science, 22, 1095-1100. doi: 10.1177/0956797611417262

Oreopoulos, P., \& Salvanes, K. G. (2011). Priceless: The non pecuniary benefits of schooling. Journal of Economic Perspectives, 25(1), 159-184. doi: 10.1257/0895330 11798837765

Pardo, A., \& San Martín, R. (1994). Análisis de datos en Psicología. Madrid, España: Ediciones Pirámide.

Rojas, M. (2016). Happiness, Research, and Latin America. En Mariano Rojas (Ed.), Handbook of Happiness Research in Latin America (pp. 1-13). Dordrecht, Holanda: Springer. doi: 10.1007/978-94-017-7203-7

Salinas-Jiménez, M., Artés, J., \& Salinas-Jiménez, J. (2013). How do educational attainment and occupational and wage-earner statuses affect life satisfaction? A gender perspective study. Journal of Happiness Studies, 14(2), 367-388. doi: 10.1007/s10902-012-9334-6

Santamaría, P. (2014). Bienestar subjetivo en el ámbito rural. (Tesis de Grado). Universidad del País Vasco, Bilbao, España.
Seligman, M. (2009). In a search of happiness. How to enjoy life everyday. Moscú, Rusia: Mann, Ivanov and Ferber.

Seligman, M. E., Parks, A. C., \& Steen, T. (2004). A balanced psychology and a full life. Philosophical TransactionsRoyal Society of London Series B Biological Sciences 359(1449), 1379-1381. doi: 10.1098/rstb.2004.1513

Steel, P., Schmidt, J., \& Shultz, J. (2008). Refining the relationship between personality and subjective wellbeing. Psychological Bulletin, 134, 138-161. doi: 10.10 37/0033-2909.134.1.138

Stiglitz, J. E. (2012). The price of inequality How today's divided society endangers our future. Nueva York, Estados Unidos de América: W. W. Norton.

Susniene, D., \& Jurkauskas, A. (2015). The concepts of quality of life and happiness-correlation and differences. Engineering Economics, 63(4), 58-66.

Toribio, L., González, N. I., Valdez, J. L., González, S., \& Oudhof, B. H. (2012). Validación de la escala de felicidad de Alarcón para adolescentes mexicanos. Psicología Iberoamericana, 20(1), 71-79.

Veenhoven, R. (1998). Calidad de vida y felicidad: No es exactamente lo mismo. En G. De Girolamo (Ed.), Salud y calidad de vida (pp. 67-95). Roma, Italia: II Pensamiento Científico.

Veenhoven, R. (2015). Social conditions for human happiness: A review of research. International Journal of Psychology, 50(5), 379-391. doi: 10.1002/ijop.12161

Velásquez, L. (2016). The Importance of Relational Goods for Happiness: Evidence from Manizales, Colombia. En Mariano Rojas (Ed.), Handbook of Happiness Research in Latin America (pp. 91-112). Dordrecht, Holanda: Springer. doi: 10.1007/978-94-017-7203-7

Velicer, W. F., \& Fava, J. L. (1998). Affects of variable and subject sampling on factor pattern recovery. Psychological methods, 3(2), 231-251. doi: 10.1037/10 82-989X.3.2.231

Vittersø, J., \& Nilsen, F. (2002). The conceptual and relational structure of subjective well-being, neuroticism, and extraversion: once again, neuroticism is the important predictor of happiness. Social Indicators Research, 57, 89-118.doi: 10.1023/A:1 013831602280 
Wilkinson, R. G., \& Pickett, K. E. (2009). Income inequality and social dysfunction. Annual Review of Sociology, 35, 493-511. doi: 10.1146/annurev-soc-070308-115926

Yamamoto, J. (2016). The Social Psychology of Latin American Happiness. En Mariano Rojas (Ed.), Handbook of Happiness Research in Latin America (pp. 31-49). Dordrecht, Holanda: Springer. doi: 10.1007/ 978-94-017-7203-7

Zubieta, E., Muratori, M., \& Fernandez, O. (2012). Bienestar subjetivo y psicosocial: explorando diferencias de género. Salud \& Sociedad, 3(1), 66-76.

\section{Sofía Esmeralda Auné}

Instituto de Investigaciones, Facultad de Psicología, Universidad de Buenos Aires, Argentina; Consejo Nacional de Investigaciones Científicas y Técnicas (CONICET)

Lic. y Prof. en Enseñanza Media y Superior en Psicología. Facultad de Psicología, Universidad de Buenos Aires (FP - UBA), Argentina. Cursando estudios de Doctorado en FP - UBA con beca del Consejo Nacional de Investigaciones Científicas y Técnicas (CONICET). Ayudante de Trabajos Prácticos en la asignatura Estadística Cátedra Segunda de la FP - UBA.

Autor corresponsal: sofiaaune177@hotmail.com

Facundo Juan Pablo Abal

Instituto de Investigaciones, Facultad de Psicología, Universidad de Buenos Aires, Argentina; Consejo Nacional de Investigaciones Científicas y Técnicas (CONICET)

Dr. en Psicología. Lic. en Psicología. FP - UBA. Investigador Asistente del CONICET. Profesor Adjunto en la asignatura Estadística Cátedra Segunda de la FP - UBA. Jefe de Trabajos Prácticos en la asignatura Estadística Cátedra Primera de la FP - UBA. Autor de numerosos artículos científicos de psicología en revistas indizadas.

afjp79@hotmail.com

\section{Horacio Félix Attorresi}

Instituto de Investigaciones, Facultad de Psicología, Universidad de Buenos Aires, Argentina

Licenciado en Ciencias Matemáticas. Facultad de Ciencias Exactas y Naturales. Universidad de Buenos Aires. Profesor Titular Consulto en la asignatura Estadística Cátedra Segunda de la FP - UBA. Autor de numerosos artículos científicos de psicología en revistas indizadas. Ha dirigido diversos proyectos de investigación en Psicología.

horacioattorresi@gmail.com

La investigación que se presenta en este artículo fue realizada con subsidios de la Universidad de Buenos Aires UBACyT 2014-17 Código Nº 20020130100320BA y de la Agencia Nacional de Promoción Científica y Tecnológica ANPCyT PICT 2011-0826. 